\title{
Gravitational and Electromagnetic Field of an Isolated Rotating Charged Particle
}

\author{
Bikash Kumar Borah \\ Department of Physics, Jorhat Institute of Science and Technology, Jorhat, India \\ Email address: \\ bikashgargilisa@gmail.com
}

\section{To cite this article:}

Bikash Kumar Borah. Gravitational and Electromagnetic Field of an Isolated Rotating Charged Particle. International Journal of Applied Mathematics and Theoretical Physics. Vol. 7, No. 1, 2021, pp. 16-27. doi: 10.11648/j.ijamtp.20210701.13

Received: February 18, 2021; Accepted: March 3, 2021; Published: March 12, 2021

\begin{abstract}
A charged isolated particle with spherically symmetry is considered at origin in empty space. The particle has both mass and charge; therefore it is under the influence of both gravitational and electro-magnetic field. So to find out a line element especial attention is given in Einstein's gravitational and Maxwell's electro-magnetic field equations. Initially Einstein's field equations are considered individually for gravitational and electro-magnetic fields in empty space. In this work initially starts with Schwarzschild like solution and then a simple elegant, systematic method is used. In these methods the e-m field tensor is also used from Maxwell's electro-magnetic field equations. Finally thus a new metric is found for both positive and negative charged particles. The new metric for electron is not same as the metric is devised by Reissner and Nordstrom. The new metric for proton is used to find another new metric for rotating charged particle. The new metric is extended for the massive body and gives us some new information about the mass required to stop electro-magnetic interaction. It gives interesting information that planet having mass more than 1.21 times of Jupiter mass, live cannot survive. Also gives information that the mass greater than the aforesaid mass there is no electrically charged body in the universe.
\end{abstract}

Keywords: Line Element, Metric, Gravitational Field, e-m Field, e-m Field Tensor

\section{Introduction}

Einstein's field equations [1] are a set of nonlinear differential equations, so these equations are difficult to find the exact analytical solution. The exact solution has got physical meanings only in case of some simplified assumptions; among these solutions which are Schwarzschild solution [2], Kerr's solution [3, 4] and FRW models [5-8] for cosmology. T Kaluza [9] in 1921 and later Oskar Klein [10, 11] in 1926 try to solve the relativity as a geometrical theory of gravitation and electro-magnetic (e-m) field. The gravitational field due to an isolated electron was given by Reissner [12, 13] and Gunner Nordstrom [14] in 1921 and later by G. B. Jeffery [15]. Schwarzschild metric was understood to describe a black hole [16] in the year 1958 and Kerr $[3,17]$ generalised the solution for rotating black hole in the year 1963. Newman $[18,19]$ try to describe the metric for charged, rotating body on the basis of Reissner-Nordstrom solution in the year 1965 .

Today different authors [20-26] proposed different methods of solution of Einstein field equations. A brief introduction is given here about Schwarzschild, ReissnerNordstrom, Kerr and Newman metrics.

\subsection{Schwarzschild Metric}

The original field equation of Einstein's in empty space is given by

$$
R_{\mu v}=0
$$

The solution of the above Einstein's field equations in empty space of an isolated particle continually at rest at the origin was first given by Schwarzschild and the metric is

$$
d s^{2}=-\left(1+\frac{A}{r}\right)^{-1} d r^{2}-r^{2} d \theta^{2}-r^{2} \sin ^{2} \theta d \varphi^{2}+\left(1+\frac{A}{r}\right) d t^{2}
$$

In above equation Schwarzschild put the integrating constant $A=-2 m$. This was done by Schwarzschild in order to facilitate the physical interpretation of $m$ as the mass of the gravitating particle. Hence, 


$$
d s^{2}=-\left(1-\frac{2 m}{r}\right)^{-1} d r^{2}-r^{2} d \theta^{2}-r^{2} \sin ^{2} \theta d \varphi^{2}+\left(1-\frac{2 m}{r}\right) d t^{2}
$$

Schwarzschild took $m=\frac{G M}{c^{2}}$ in the above (3) and then in ordinary units,

$$
d s^{2}=-\left(1-\frac{2 G M}{r c^{2}}\right)^{-1} d r^{2}-r^{2} d \theta^{2}-r^{2} \sin ^{2} \theta d \varphi^{2}+\left(1-\frac{2 G M}{r c^{2}}\right) c^{2} d t^{2}
$$

This metric is spherically symmetric and may be regarded as the gravitational field of a non-rotating point mass $M$ at rest at origin.
The metric for a non-rotating isolated electron was given by Reissner and Gunner Nordstrom as,

\subsection{Reissner-Nordstrom Metric}

$$
d s^{2}=-\left(1-\frac{2 m}{r}+\frac{4 \pi \varepsilon^{2}}{r^{2}}\right)^{-1} d r^{2}-r^{2} d \theta^{2}-r^{2} \sin ^{2} \theta d \varphi^{2}+\left(1-\frac{2 m}{r}+\frac{4 \pi \varepsilon^{2}}{r^{2}}\right) d t^{2}
$$

Equation (5) gives the Newtonian potential $\phi$ given as,

$$
\varphi=-\frac{m}{r}+\frac{2 \pi \varepsilon^{2}}{r^{2}}
$$

Furthermore the potential force is,

$$
\frac{\partial \phi}{\partial r}=\frac{m}{r^{2}}-\frac{4 \pi \varepsilon^{2}}{r^{3}}
$$

In equation (6) if we consider $m=0$ then the force is inversely proportional to the cube of the distance, which is impossible. This means that there may have some discrepancy in mathematical derivation process in the
Reissner-Nordstrom metric. This compelled me to think this problem seriously. The author $[25,26]$ is trying to solve this problem but still no satisfaction. Again considering this problem a more simple elegant and systematic method is used to modify the new metric which is derived in this research paper. Later taking this new metric of non-rotating charged body a more enlighten way to find a solution for rotating charged body is attempted.

\subsection{Kerr Metric}

The Kerr solution in Boyer-Lindquist coordinates [3, 17, 19] is

$$
\begin{aligned}
d s^{2}= & -\left(1-\frac{2 m r}{\rho^{2}}\right) d t^{2}-\frac{4 m r a \sin ^{2} \theta}{\rho^{2}} d \varphi d t+\frac{\rho^{2}}{\Delta} d r^{2}+\rho^{2} d \theta^{2} \\
& +\left(\mathrm{r}^{2}+a^{2}+\frac{2 m r a^{2} \sin ^{2} \theta}{\rho^{2}}\right) \sin ^{2} \theta d \varphi^{2}
\end{aligned}
$$

Equivalent forms are

$$
d s^{2}=-\frac{\Delta}{\rho^{2}}\left[d t-a \sin ^{2} \theta d \varphi\right]^{2}+\frac{\rho^{2}}{\Delta} d r^{2}+\rho^{2} d \theta^{2}+\frac{\sin ^{2} \theta}{\rho^{2}}\left[\left(r^{2}+a^{2}\right) d \varphi-a d t\right]^{2}
$$

And

$$
d s^{2}=-d t^{2}+\frac{2 m r}{\rho^{2}}\left[d t-a \sin ^{2} \theta d \varphi\right]^{2}+\frac{\rho^{2}}{\Delta} d r^{2}+\rho^{2} d \theta^{2}+\left(r^{2}+a^{2}\right) \sin ^{2} \theta d \varphi^{2}
$$

In the above equations considered,

$$
\rho^{2}=r^{2}+a^{2} \cos ^{2} \theta, \Delta=r^{2}+a^{2}-2 m r \text { and } c=1
$$

\subsection{Newman Metric}

The metric for rotating charged particle according to Kerr-Newman [19] solution is 


$$
d s^{2}=-\frac{\Delta}{\rho^{2}}\left[d t-a \sin ^{2} \theta d \varphi\right]^{2}+\frac{\rho^{2}}{\Delta} d r^{2}+\rho^{2} d \theta^{2}+\frac{\sin ^{2} \theta}{\rho^{2}}\left[\left(r^{2}+a^{2}\right) d \varphi-a d t\right]^{2}
$$

Equivalent form is

$$
\begin{aligned}
d s^{2}= & -\left(1-\frac{2 m r}{\rho^{2}}\right) d t^{2}-\frac{4 m r a \sin ^{2} \theta}{\rho^{2}} d \varphi d t+\frac{\rho^{2}}{\Delta} d r^{2}+\rho^{2} d \theta^{2} \\
& +\left(\mathrm{r}^{2}+a^{2}+\frac{2 m r a^{2} \sin ^{2} \theta}{\rho^{2}}\right) \sin ^{2} \theta d \varphi^{2}
\end{aligned}
$$

In these above equations $\rho^{2}=r^{2}+a^{2} \cos ^{2} \theta, c=1$, but $\Delta=r^{2}+a^{2}-2 m r+q^{2}$. The symbol $q$ is taken as the charge of the particle.

\section{Derivation of the New Metric for Non-rotating Charged Particle}

A simple elegant and systematic method is used here to determine the metric for non-rotating isolated charged body. Let us consider an isolated proton which is positively charged is placed at origin at rest in empty space. The proton has both gravitational and e-m field since it has both mass and charge. The gravitational and e-m fields of the proton are assumed to be spherically symmetric. The interaction range of the gravitational field is from infinity to $10^{-33} \mathrm{~cm}$ and for e$\mathrm{m}$ field it is from infinity to $10^{-8} \mathrm{~cm}$; therefore both gravitational and e-m fields have the interaction range from infinity to $10^{-8} \mathrm{~cm}$. Since the proton is under the influence of both gravitational and e-m fields in empty space, hence Einstein field equation (1) is applicable.

The fundamental metric or line element is given by,

$$
d s^{2}=g_{\mu v} d x^{\mu} d x^{v}
$$

The most general form of the metric or line element for gravitational field of an isolated proton at the origin at rest in empty space satisfying the condition of spherically symmetric can be written as,

$$
d s^{2}=-e^{\lambda} d r^{2}-r^{2} d \theta^{2}-r^{2} \sin ^{2} \theta d \varphi^{2}+e^{v} d t^{2}
$$

Here $\lambda$ and $v$ are the functions of $r$ only.

Similarly the most general form of the metric for the e-m field of an isolated proton at origin at rest in empty space satisfying the condition of spherically symmetric is given by,

$$
d s^{2}=-e^{a} d r^{2}-r^{2} d \theta^{2}-r^{2} \sin ^{2} \theta d \varphi^{2}+e^{b} d t^{2}
$$

In this above equation $a$ and $b$ are the functions of $r$ only.

The equations (14) and (15) are individual metric or line element for each field of the isolated proton at origin at rest. Actually the isolated proton at rest at origin is under the influence of both self-gravitational and self-electromagnetic fields together. Therefore the most general form of metric or line element is,

$$
d s^{2}=-e^{a} e^{\lambda} d r^{2}-r^{2} d \theta^{2}-r^{2} \sin ^{2} \theta d \varphi^{2}+\mathrm{e}^{b} \mathrm{e}^{v} d t^{2}
$$

Or we can write as,

$$
d s^{2}=-e^{\rho} d r^{2}-r^{2} d \theta^{2}-r^{2} \sin ^{2} \theta d \varphi^{2}+\mathrm{e}^{\sigma} d t^{2}
$$

Where $\rho=\lambda+a$ and $\sigma=v+\mathrm{b}$

The solution of (14) was given by Schwarzschild as,

$$
d s^{2}=-\left(1-\frac{2 m}{r}\right)^{-1} d r^{2}-r^{2} d \theta^{2}-r^{2} \sin ^{2} \theta d \varphi^{2}+\left(1-\frac{2 m}{r}\right) d t^{2}
$$

The relation between $m$ and the proton mass $m_{p}$ is,

$$
m=\frac{G m_{p}}{c^{2}}
$$

Again the solution of (15) becomes,

$$
d s^{2}=-\left(1+\frac{B}{r}\right)^{-1} d r^{2}-r^{2} d \theta^{2}-r^{2} \sin ^{2} \theta d \varphi^{2}+\left(1+\frac{B}{r}\right) d t^{2}
$$

In above equation the integrating constant is put as $B$.

Similarly the solution of (17) we can find out the solution as, 


$$
d s^{2}=-\left(1+\frac{D}{r}\right)^{-1} d r^{2}-r^{2} d \theta^{2}-r^{2} \sin ^{2} \theta d \varphi^{2}+\left(1+\frac{D}{r}\right) d t^{2}
$$

Here we have taken $D$ as an integrating constant.

But still the value of $B$ and $D$ are unknown to us. To find out the value of $B$ let we first find out an equation of motion of a particle [26] with very low velocity in static field, and then we find the e-m field tensor [26] and finally find out the value of $B$. Then we are able to find out the value of $D$.

\subsection{Equation of Motion of a Particle}

Let we consider a particle is in motion with very low velocity in a static field. Therefore the geodesic equation for a non-relativistic particle is:

$$
\frac{d^{2} x^{\alpha}}{d s^{2}}+\Gamma_{\mu v}^{\alpha} \frac{d x^{\mu}}{d s} \frac{d x^{v}}{d s}=0
$$

The metric in Riemannian space is given by the (13) and let us now assume that in (13), $g_{\mu v}$ are not constant, but differ from the values in flat space by infinitesimal amount. Therefore we can write,

$$
g_{\mu v}=\xi_{\mu v}+\eta_{\mu v}
$$

Putting $\mu=\rho$ and $v=\alpha$ in above equation

$$
g_{\rho \alpha}=\xi_{\rho \alpha}+\eta_{\rho \alpha}
$$

In above equation $\eta_{\mu v}$ are very small quantities and functions of $x, y, z$; but independent of time $t$. This means that,

$$
\frac{\partial \eta_{\mu v}}{\partial x^{4}}=\frac{\partial g_{\mu v}}{\partial x^{4}}=0
$$

Furthermore, the relation between Christofell's symbols of second kinds with first kinds,

$$
\Gamma_{\mu \nu}^{\alpha}=g^{\rho \alpha} \Gamma_{\rho, \mu v}
$$

Then we can write from (23) in covariance form

$$
\Gamma_{\mu \nu}^{\alpha}=\frac{1}{2}\left(\xi^{\rho \alpha}+\eta^{\rho \alpha}\right)\left(\frac{\partial \eta_{\alpha \mu}}{\partial x^{\nu}}+\frac{\partial \eta_{\alpha \nu}}{\partial x^{\mu}}-\frac{\partial \eta_{\mu v}}{\partial x^{\alpha}}\right)
$$

Neglected $\eta^{\rho \alpha}$, because it is very small quantity and putting $\rho=\alpha$ we obtain,

$$
\Gamma_{\mu \nu}^{\alpha}=\frac{1}{2}\left(\frac{\partial \eta_{\alpha \mu}}{\partial x^{\nu}}+\frac{\partial \eta_{\alpha v}}{\partial x^{\mu}}-\frac{\partial \eta_{\mu \nu}}{\partial x^{\alpha}}\right)
$$

Taking as $x^{1}=x, x^{2}=y, x^{3}=z$ and $x^{4}=c t$ we can write,

$$
d s^{2}=-d x^{2}-d y^{2}-d z^{2}+c^{2} d t^{2}
$$

This gives,

$$
d s^{2}=-\mathrm{v}^{2} d t^{2}+c^{2} d t^{2}=c^{2} d t^{2}\left(1-\frac{\mathrm{v}^{2}}{c^{2}}\right),
$$

But the velocity $\mathrm{v}$ is non-relativistic and hence $\mathrm{v} \ll<c$ then it gives,

$$
d s=c d t=d x^{4}
$$

So we can write,

$$
\frac{d x^{1}}{d s}=\frac{d x^{2}}{d s}=\frac{d x^{3}}{d s}=0 \text { and } \frac{d x^{4}}{d s}=1
$$

Therefore by virtue of (22) and using (25) the above equation yields

$$
\frac{d^{2} x^{\alpha}}{d s^{2}}=-\Gamma_{44}^{\alpha}=-\frac{1}{2}\left(\frac{\partial \eta_{44}}{\partial x^{\alpha}}\right) \text { for } \alpha=1,2,3
$$

Again using the (26),

$$
\frac{d^{2} x^{\alpha}}{d t^{2}}=-\frac{\partial}{\partial x^{\alpha}}\left(\frac{1}{2} c^{2} g_{44}\right)
$$

This is the equation for motion of a particle.

Now Newtonian equations of motion are

$$
\frac{d^{2} x^{\alpha}}{d t^{2}}=-\frac{\partial \phi}{\partial x^{\alpha}}
$$

Where $\phi$ is gravitational potential function and $\alpha=1,2,3$.

Therefore from (29) and (30) we can write,

$$
-\frac{\partial}{\partial r}\left(\frac{1}{2} c^{2} g_{44}\right)=-\frac{\partial \phi}{\partial r}
$$

\subsection{The E-m Field Tensor}

We have found the equation of motion for a particle in static field. Now we are going to find out the e-m field tensor for a charged particle which is also required to find out the value of constant $B$. Since the field in (15) is symmetrical and our assumption implies that the field is purely electrostatic. So the magnetic field intensities are,

$$
H_{x}, H_{y}, H_{z}=0
$$

The general potential $K^{\mu}$ is defined in terms of electromagnetic potential $A$ and scalar potential $\psi$ as, 


$$
K^{\mu}=\left(-A_{x},-A_{y},-A_{z}, \psi\right)
$$

Now the e-m field tensor $F_{\mu \nu}$ is defined as,

$$
F^{\mu v}=K_{\mu, v}-K_{v, \mu}=\frac{\partial K_{\mu}}{\partial x^{v}}-\frac{\partial K_{v}}{\partial x^{\mu}}
$$

And $\vec{H}=\nabla \times \vec{A}$

In the view of this, (32) gives

$$
A_{x}, A_{y}, A_{z}=0
$$

This means that $\psi$ is a function of $r$ only.

Therefore using the (33) we can write

$$
F_{12}, F_{23}, F_{31}, F_{24}, F_{34}=0 \text { and } F_{14}=-\frac{\partial \psi}{\partial r}
$$

This implies that the only non-vanishing components of

$$
F_{\mu \nu} \text { is } F_{14} \text { and } F_{14}=-F_{41} \text {. }
$$

The current density $J^{\mu}$ can be written as,

$$
J^{\mu}=F_{v}^{\mu v}=\frac{\partial F^{\mu v}}{\partial x^{v}}+F^{\alpha v} \Gamma_{\alpha \nu}^{\mu}+F^{\mu \alpha} \Gamma_{\alpha \nu}^{v}
$$

The value of $F^{\alpha v} \Gamma_{\alpha \nu}^{\mu}=0$ and taking $J^{\mu}=q$, where $q$ is considered as charge, this yield

$$
\sqrt{-g} q=\frac{\partial\left(\sqrt{-g} F^{41}\right)}{\partial r}
$$

But there is no charge and no current in the space surrounding the charged particle which is at origin. Therefore,

$$
\frac{\partial\left(\sqrt{-g} F^{41}\right)}{\partial r}=0
$$

Furthermore,

$$
F^{41}=-F_{41}=-F^{14}=F_{14}=-\frac{\partial \psi}{\partial r}
$$

From the above equations the (36) can be written as,

$$
\frac{\partial}{\partial r}\left[e^{(a+b) / 2} r^{2} \sin \theta\left(-e^{-(a+b)}\right) F_{41}\right]=0
$$

Integrating the above equation we get

$$
F_{14}=\frac{\varepsilon}{r^{2}} e^{(a+b) / 2}
$$

The symbol $\varepsilon$ is considered as an absolute constant. The constant $\varepsilon$ is related with the charge of the particle which is situated at origin. This means $4 \pi \varepsilon(=q)$ is charge.
After rigorous calculations of the (15) gives us $b=-a$, putting this in (37) and compare with (34) we get,

$$
-\frac{\partial \psi}{\partial r}=\frac{\varepsilon}{r^{2}}
$$

\subsection{The Value of Constant B}

Suppose

$$
-\frac{\partial \phi}{\partial r}=-\frac{\partial \psi}{\partial r}
$$

Then (38) comparing with (31) we get,

$$
-\frac{\partial}{\partial r}\left(\frac{1}{2} c^{2} g_{44}\right)=\frac{\varepsilon}{r^{2}}
$$

Integrating above equation,

$$
g_{44}=\frac{2 \varepsilon}{r c^{2}}+k
$$

In the above equation $k$ is considered as an integrating constant.

At $r \rightarrow \infty, g_{44}=1$ gives $k=1$, hence

$$
g_{44}=e^{b}=\left(1+\frac{2 \varepsilon}{r c^{2}}\right)
$$

The above equation compare with the time coefficient of the (20) i.e.

$$
g_{44}=e^{b}=\left(1+\frac{B}{r}\right)
$$

Gives,

$$
B=\frac{2 \varepsilon}{c^{2}}
$$

\subsection{The New Metric}

We know from (2) and (3),

$$
e^{v}=\left(1+\frac{A}{r}\right)=\left(1-\frac{2 m}{r}\right)=\left(1-\frac{2 G m_{p}}{r c^{2}}\right)
$$

Considering $4 \pi \varepsilon=q$, the charge of the particle and $\frac{1}{4 \pi}=K$, is a constant then in (20)

$$
e^{b}=\left(1+\frac{B}{r}\right)=\left(1+\frac{2 \varepsilon}{r c^{2}}\right)=\left(1+\frac{2 K q}{r c^{2}}\right)
$$

Putting (44) and (45) in the time coefficient of (16) or (17) 


$$
e^{\sigma}=e^{v} e^{b}=\left(1+\frac{A}{r}\right)\left(1+\frac{B}{r}\right)=\left(1-\frac{2 G m_{p}}{r c^{2}}\right)\left(1+\frac{2 K q}{r c^{2}}\right)
$$

This gives,

$$
e^{\sigma}=\left(1+\frac{A}{r}+\frac{B}{r}+\frac{A B}{r^{2}}\right)=\left(1-\frac{2 G m_{p}}{r c^{2}}+\frac{2 K q}{r c^{2}}-\frac{4 G m_{p} K q}{r^{2} c^{4}}\right)
$$

The last term of r. h. s. of above equation is tends to zero since higher terms of $r$ and $c$ are in the denominator. Therefore

This gives,

$$
d s^{2}=-\left(1-\frac{2 G m_{p}}{r c^{2}}+\frac{2 K q}{r c^{2}}\right)^{-1} d r^{2}-r^{2} d \theta^{2}-r^{2} \sin ^{2} \theta d \varphi^{2}+\left(1-\frac{2 G m_{p}}{r c^{2}}+\frac{2 K q}{r c^{2}}\right) d t^{2}
$$

From (47),

$$
g_{\mu v}=\left[\begin{array}{cccc}
-\left(1-\frac{2 G m_{p}}{r c^{2}}+\frac{2 K q}{r c^{2}}\right)^{-1} & 0 & 0 & 0 \\
0 & -r^{2} & 0 & 0 \\
0 & 0 & -r^{2} \sin ^{2} \theta & 0 \\
0 & 0 & 0 & \left(1-\frac{2 G m_{p}}{r c^{2}}+\frac{2 K q}{r c^{2}}\right)
\end{array}\right]
$$

For negatively charged particle or electron,

$$
d s^{2}=-\left(1-\frac{2 G m_{e}}{r c^{2}}-\frac{2 K q}{r c^{2}}\right)^{-1} d r^{2}-r^{2} d \theta^{2}-r^{2} \sin ^{2} \theta d \varphi^{2}+\left(1-\frac{2 G m_{e}}{r c^{2}}-\frac{2 K q}{r c^{2}}\right) d t^{2}
$$

In above equation $m_{e}$ is the mass of electron.

\section{Derivation of the New Metric for Rotating Charged Particle}

We consider the (47) and taking $m_{p}=M$ and $q=Q$ for a massive body,

$$
d s^{2}=-\left(1-\frac{2 G M}{r c^{2}}+\frac{2 K Q}{r c^{2}}\right)^{-1} d r^{2}-r^{2} d \theta^{2}-r^{2} \sin ^{2} \theta d \varphi^{2}+\left(1-\frac{2 G M}{r c^{2}}+\frac{2 K Q}{r c^{2}}\right) d t^{2}
$$

Equation (50) can be written in other form as

$$
d s^{2}=-\left(1-\frac{2 G M}{r c^{2}}+\frac{2 K Q}{r c^{2}}\right) d t^{2}+\left(1-\frac{2 G M}{r c^{2}}+\frac{2 K Q}{r c^{2}}\right)^{-1} d r^{2}+r^{2} d \theta^{2}+r^{2} \sin ^{2} \theta d \varphi^{2}
$$

Or

$$
d s^{2}=-\left(1-\frac{r_{S}}{r}+\frac{r_{Q}}{r}\right) d t^{2}+\left(1-\frac{r_{S}}{r}+\frac{r_{Q}}{r}\right)^{-1} d r^{2}+r^{2} d \theta^{2}+r^{2} \sin ^{2} \theta d \varphi^{2}
$$

Here 


$$
r_{S}=\frac{2 G M}{c^{2}}, r_{Q}=\frac{2 K Q}{c^{2}}
$$

Now we consider the constants are simply in natural units as $c=G=K=1$ and which gives $r_{S}=2 M, r_{Q}=2 Q$. Therefore we can write,

$$
d s^{2}=-\left(1-\frac{2 M}{r}+\frac{2 Q}{r}\right) d t^{2}+\left(1-\frac{2 M}{r}+\frac{2 Q}{r}\right)^{-1} d r^{2}+r^{2} d \theta^{2}+r^{2} \sin ^{2} \theta d \varphi^{2}
$$

Now we can transform (53) in to a null co-ordinate system where the time co-ordinate $t$ is replaced by the null time coordinate $u$ as,

$$
u=t+r+(2 M-2 Q) \ln \left\{\frac{r}{(2 M-2 Q)}-1\right\}
$$

Therefore

$$
d u=d t+\left(1-\frac{2 M}{r}+\frac{2 Q}{r}\right)^{-1} d r
$$

The equation (55) putting in (53) we get

$$
d s^{2}=-\left(1-\frac{2 M}{r}+\frac{2 Q}{r}\right) d u^{2}+2 d u d r+r^{2} d \theta^{2}+r^{2} \sin ^{2} \theta d \varphi^{2}
$$

Therefore,

$$
g_{\mu \nu}=\left(\begin{array}{cccc}
-\left(1-\frac{2 M}{r}+\frac{2 Q}{r}\right) & 1 & 0 & 0 \\
1 & 0 & 0 & 0 \\
0 & 0 & r^{2} & 0 \\
0 & 0 & 0 & r^{2} \sin ^{2} \theta
\end{array}\right)
$$

The contra-variant form of $g_{\mu v}$ is

$$
g^{\mu \nu}=\left(\begin{array}{cccc}
0 & 1 & 0 & 0 \\
1 & \left(1-\frac{2 M}{r}+\frac{2 Q}{r}\right) & 0 & 0 \\
0 & 0 & \frac{1}{r^{2}} & 0 \\
0 & 0 & 0 & \frac{1}{r^{2} \sin ^{2} \theta}
\end{array}\right)
$$

The metric can now be expressed in terms of null tetrad $\{l, n, m, \bar{m}\}$. The vectors $l, n$ are real while $m, \bar{m}$ are complex conjugates. The world space metric is

$$
g_{\mu v}=-l_{\mu} n_{v}-l_{v} n_{\mu}+m_{\mu} \bar{m}_{v}+m_{v} \bar{m}_{\mu}
$$

The contra-variant form is

$$
g^{\mu v}=-l^{\mu} n^{v}-l^{v} n^{\mu}+m^{\mu} \bar{m}^{v}+m^{v} \bar{m}^{\mu}
$$

The equivalent null tetrads are 


$$
\left.\begin{array}{l}
l^{\mu}=(0,1,0,0)=\partial r \\
n^{\mu}=\left(-1,-\frac{1}{2}\left(1-\frac{2 M}{r}+\frac{2 Q}{r}\right), 0,0\right)=-\partial u-\frac{1}{2}\left(1-\frac{2 M}{r}+\frac{2 Q}{r}\right) \partial r \\
m^{\mu}=\frac{1}{r \sqrt{2}}\left(0,0,1, \frac{i}{\sin \theta}\right)=\frac{1}{r \sqrt{2}}(\partial \theta+i \operatorname{cosec} \theta \partial \varphi) \\
\bar{m}^{\mu}=\frac{1}{r \sqrt{2}}\left(0,0,1,-\frac{i}{\sin \theta}\right)=\frac{1}{r \sqrt{2}}(\partial \theta-i \operatorname{cosec} \theta \partial \varphi)
\end{array}\right\}
$$

We can easily found the followings:

$$
\left.\begin{array}{l}
g^{00}=0, \quad g^{01}=g^{10}=1 \\
g^{11}=\left(1-\frac{2 M}{r}+\frac{2 Q}{r}\right), \quad g^{22}=\frac{1}{r^{2}} \\
g^{33}=\frac{1}{r^{2} \sin ^{2} \theta} \text { and } g^{12}=g^{13}=g^{23}=g^{31}=\ldots=0
\end{array}\right\}
$$

The used the method used by Newman and Janis [4], to make $r$ complex (with $\bar{r}$ its complex conjugate) and replace the tetrad above by,

$$
\begin{aligned}
& l^{\mu}=\left(\begin{array}{lll}
0, & 1, & 0,0
\end{array}\right)=\partial r \\
& n^{\mu}=\left(-1,-\frac{1}{2}+\frac{M}{2}\left(\frac{1}{r}+\frac{1}{\bar{r}}\right)-\frac{Q}{2}\left(\frac{1}{r}+\frac{1}{\bar{r}}\right), 0,0\right)=-\partial u-\frac{1}{2} M^{\prime} \partial r \\
& m^{\mu}=\frac{1}{\bar{r} \sqrt{2}}\left(0,0,1, \frac{i}{\sin \theta}\right)=\frac{1}{\bar{r} \sqrt{2}}(\partial \theta+i \operatorname{cosec} \theta \partial \varphi) \\
& \bar{m}^{\mu}=\frac{1}{r \sqrt{2}}\left(0,0, \quad 1,-\frac{i}{\sin \theta}\right)=\frac{1}{r \sqrt{2}}(\partial \theta-i \operatorname{cosec} \theta \partial \varphi)
\end{aligned}
$$

Where $M^{\prime}=\left(1-\frac{M}{r}-\frac{M}{\bar{r}}+\frac{Q}{r}+\frac{Q}{\bar{r}}\right)$

Still the vectors $l^{\mu}, n^{\mu}$ are real and $m^{\mu}, \bar{m}^{\mu}$ are complex conjugates of each other. Now perform the transformations as

$$
\left.\begin{array}{l}
r=r^{\prime}-i a \cos \theta, \\
u=u^{\prime}-i a \cos \theta \\
\theta=\theta^{\prime} \\
\varphi=\varphi^{\prime}
\end{array}\right\}
$$

Here $r^{\prime}$ and $u^{\prime}$ real. The ' $a$ ' is a parameter and later we shall justify its interpretation as the angular momentum of the body. For determination the values of $m^{\prime \mu}$ and $\bar{m}^{\prime \mu}$ we used the usual formula given below:

$$
V^{\prime \mu}=\frac{\partial x^{\prime \mu}}{\partial x^{\nu}} V^{v}
$$

Using the above formula the values of,

$$
\begin{gathered}
m^{\prime 0}=\frac{-i a \sin \theta}{\bar{r} \sqrt{2}}, m^{\prime 1}=\frac{-i a \sin \theta}{\bar{r} \sqrt{2}}, m^{\prime 2}=\frac{1}{\bar{r} \sqrt{2}} \text { and } m^{\prime 3}=\frac{i}{\bar{r} \sqrt{2} \sin \theta} \\
\bar{m}^{\prime 0}=\frac{i a \sin \theta}{r \sqrt{2}}, \bar{m}^{\prime 1}=\frac{i a \sin \theta}{r \sqrt{2}}, \bar{m}^{\prime 2}=\frac{1}{r \sqrt{2}} \text { and } \bar{m}^{\prime 3}=-\frac{i}{r \sqrt{2} \sin \theta}
\end{gathered}
$$


Using (64), (65) and (66) the tetrad becomes,

$$
\left.\begin{array}{l}
l^{\prime \mu}=\left(\begin{array}{lll}
0,1, & 0,0
\end{array}\right) \\
n^{\prime \mu}=\left(\begin{array}{lll}
-1, & -\frac{1}{2}+\frac{(M-Q) r^{\prime}}{\left(r^{\prime 2}+a^{2} \cos ^{2} \theta\right)}, 0,0
\end{array}\right) \\
m^{\prime \mu}=\frac{1}{\left(r^{\prime}+i a \cos \theta\right) \sqrt{2}}\left(-i a \sin \theta,-i a \sin \theta, 1, \frac{i}{\sin \theta}\right) \\
\bar{m}^{\prime \mu}=\frac{1}{\left(r^{\prime}-i a \cos \theta\right) \sqrt{2}}\left(i a \sin \theta, i a \sin \theta, \quad 1,-\frac{i}{\sin \theta}\right)
\end{array}\right\}
$$

Now the contravariant components of the metric using (60) and (67) and dropping the primes we get,

$$
\left.\begin{array}{l}
g^{00}=\frac{a^{2} \sin ^{2} \theta}{R^{2}}, g^{01}=g^{10}=\frac{r^{2}+a^{2}}{R^{2}}, g^{03}=g^{30}=-\frac{a}{R^{2}} \\
g^{13}=g^{31}=-\frac{a}{R^{2}}, g^{11}=\frac{r^{2}+a^{2}-2(M-Q) r}{R^{2}} \\
g^{22}=\frac{1}{R^{2}}, g^{33}=\frac{1}{R^{2} \sin ^{2} \theta} \\
g^{20}=g^{02}=g^{21}=g^{12}=g^{23}=g^{32}=0
\end{array}\right\}
$$

Where we take

$$
R^{2}=r^{2}+a^{2} \cos ^{2} \theta
$$

Therefore we can write

$$
g^{\mu \nu}=\left(\begin{array}{cccc}
\frac{a^{2} \sin ^{2} \theta}{R^{2}} & \frac{r^{2}+a^{2}}{R^{2}} & 0 & -\frac{a}{R^{2}} \\
\frac{r^{2}+a^{2}}{R^{2}} & \frac{r^{2}+a^{2}-2(M-Q) r}{R^{2}} & 0 & -\frac{a}{R^{2}} \\
0 & 0 & \frac{1}{R^{2}} & 0 \\
-\frac{a}{R^{2}} & -\frac{a}{R^{2}} & 0 & \frac{1}{R^{2} \sin ^{2} \theta}
\end{array}\right)
$$

The covariant component is

$$
g_{\mu \nu}=\left(\begin{array}{cccc}
-\left(1-\frac{2(M-Q) r}{R^{2}}\right) & 1 & 0 & -\frac{2(M-Q) r a \sin ^{2} \theta}{R^{2}} \\
1 & 0 & 0 & -a \sin ^{2} \theta \\
0 & 0 & R^{2} & 0 \\
-\frac{2(M-Q) r a \sin ^{2} \theta}{R^{2}} & -a \sin ^{2} \theta & 0 & \left(r^{2}+a^{2}+\frac{2(M-Q) r a^{2} \sin ^{2} \theta}{R^{2}}\right) \sin ^{2} \theta
\end{array}\right)
$$

Then we can write,

$$
\begin{aligned}
d s^{2}= & -\left(1-\frac{2(M-Q) r}{R^{2}}\right) d u^{2}+2 d u d r-\frac{4(M-Q) r a \sin ^{2} \theta}{R^{2}} d u d \varphi-2 a \sin ^{2} \theta d r d \varphi \\
& +R^{2} d \theta^{2}+\left(r^{2}+a^{2}+\frac{2(M-Q) r a^{2} \sin ^{2} \theta}{R^{2}}\right) \sin ^{2} \theta d \varphi^{2}
\end{aligned}
$$


Now we perform the coordinate transformations

$$
d u=d t+\frac{r^{2}+a^{2}}{\Delta} d r, d \varphi=d \psi+\frac{a}{\Delta} d r
$$

Where we take,

$$
\Delta=r^{2}+a^{2}-2(M-Q) r
$$

After doing some algebra and relabeling $\psi \rightarrow \varphi$ we can write the metric as,

$$
\begin{aligned}
d s^{2}= & -\left(1-\frac{2(M-Q) r}{R^{2}}\right) d t^{2}-\frac{4(M-Q) r a \sin ^{2} \theta}{R^{2}} d \varphi d t+\frac{R^{2}}{\Delta} d r^{2} \\
& +R^{2} d \theta^{2}+\left(r^{2}+a^{2}+\frac{2(M-Q) r a^{2} \sin ^{2} \theta}{R^{2}}\right) \sin ^{2} \theta d \varphi^{2}
\end{aligned}
$$

This is the required metric for an isolated rotating positively charged particle.

The metric tensor is

$$
g_{\mu v}=\left(\begin{array}{cccc}
-\left(1-\frac{2(M-Q) r}{R^{2}}\right) & 0 & 0 & -\frac{2(M-Q) r a \sin ^{2} \theta}{R^{2}} \\
0 & \frac{R^{2}}{\Delta} & 0 & 0 \\
0 & 0 & R^{2} & 0 \\
-\frac{2(M-Q) r a \sin ^{2} \theta}{R^{2}} & 0 & 0 & \left(r^{2}+a^{2}+\frac{2(M-Q) r a^{2} \sin ^{2} \theta}{R^{2}}\right) \sin ^{2} \theta
\end{array}\right)
$$

Here $a=(J / M)$ is the angular momentum per unit mass of the source.

\section{Results and Discussion}

In equation (75) if we consider $Q=0$ then it takes the form (7) which is Kerr solution. Again if we consider $Q=0, a=0$ then it takes the form Schwarzschild metric.

Considering (46) the Newtonian-like potential is

$$
\begin{gathered}
1-\frac{2 G m_{p}}{r c^{2}}+\frac{2 K q}{r c^{2}}=1+2 \phi \\
\phi=\frac{-G m_{p}}{r c^{2}}+\frac{K q}{r c^{2}}
\end{gathered}
$$

This gives,

$$
\frac{\partial \phi}{\partial r}=\frac{G m_{p}}{r^{2} c^{2}}-\frac{K q}{r^{2} c^{2}}
$$

If $m_{p}=0$, then the terms of r. h. s. gives,

Force $=-\frac{K q}{r^{2} c^{2}}$. This means that the force $\propto \frac{1}{r^{2}}$ i.e. the potential force in (78) satisfied the inverse square law whereas in (6) given by Reissner-Nordstrom does not satisfy the inverse square law.
Let consider the values of the constants

$$
\left.\begin{array}{l}
q=1.6 \times 10^{-19} C \\
K=9 \times 10^{18} \text { dyne }-\mathrm{cm}^{2} / C^{2} \\
G=6.670 \times 10^{-8} \text { dyne }-\mathrm{cm}^{2} / \mathrm{gm}^{2} \\
m_{p}=1.67265 \times 10^{-24} \mathrm{gm}
\end{array}\right\}
$$

Putting the above values in below:

$$
\frac{B}{2 m}=\frac{K q}{G m_{p}}=1.2907 \times 10^{31}
$$

The e-m potential is stronger than $1.29 \times 10^{31}$ times of gravitational potential.

Let us consider another proton comes near to the origin particle up to distance ' $r$ ' and interacts both electrically and gravitationally. In the above equations we have used gravitational potential energy given by Newton's law since the forces are static and weak. Therefore we have extended the Newton's law in above equations for two protons also. Consider the (46)

$$
g_{44}=\left[1-\frac{G m_{p} m_{p}}{r c^{2}}+\frac{K q^{2}}{r c^{2}}\right]
$$


In equations (81) the gravitational potential energy is very weak then e-m potential energy. Let we consider isolated particle at rest in origin is a massive body $M=N m_{p}$, $(N=1,2,3, \ldots . . \infty)$ which is nothing but the combination of protons. As number of proton increases the mass of the body increases and gravitational force increases, since all massive particles gravitationally interacts with all massive particles but one charged particle electromagnetically interacts with only one charged particle. Hence the (81) becomes,

$$
g_{44}=\left[1-\frac{1}{r c^{2}}\left(G M m_{p}-K q^{2}\right)\right]
$$

Now for flat space-time

$$
\left(K q^{2}-G M m_{p}\right)=0
$$

This gives,

$$
M=\frac{K q^{2}}{G m_{p}}
$$

Putting the values from (79) the mass required for flat space time or to stop e-m interaction is equal to $M_{e m}=2.0667735 \times 10^{12} \mathrm{gm}$.

The values of these $M_{e m}$ so large that cannot exist within the range $r\left(=10^{-8} \mathrm{~cm}\right)$. Density will be very high; hence cannot consider such massive particle. Therefore we consider $M^{\prime}\left(=\sum_{1}^{N^{\prime}} m_{p}=N^{\prime} m_{p}\right)$ such as mass $M^{\prime}$ is required to stop the e-m interaction and $R^{\prime}$ is considered as the radius of the massive body.

Now to determine the values of $M^{\prime}$ one can write,

$$
\frac{G M}{r}=\frac{G M^{\prime}}{R^{\prime}}
$$

This gives for e-m interaction

$$
M^{\prime}=M\left(\frac{R^{\prime}}{r}\right)=\frac{K q^{2}}{G m_{p}}\left(\frac{R^{\prime}}{r}\right)
$$

Number of proton contains in mass $M$ is $N\left(=M / m_{p}\right)$ and $r$ is interacting range or atomic radius then volume for $N$ atoms is

$$
V=\frac{M}{m_{p}} \times \frac{4}{3} \pi r^{3}
$$

Therefore density is

$$
\rho=\frac{M}{V}=\frac{m_{p}}{(4 / 3) \pi r^{3}}
$$

Now

$$
M^{\prime}=\frac{4}{3} \pi R^{\prime 3} \rho=m_{p}\left(\frac{R^{\prime}}{r}\right)^{3}
$$

Equating (85) with (88)

$$
\frac{R^{\prime}}{r}=\frac{1}{m_{p}}\left(\frac{K q^{2}}{G}\right)^{(1 / 2)}
$$

Putting (89) in equation (88)

$$
M^{\prime}=\left(1 / m_{p}^{2}\right)\left(\frac{K q^{2}}{G}\right)^{(3 / 2)}
$$

Using the values of $q, K, G, m_{p}$ from (79) in (90) to stop e-m interaction between two protons putting as $M^{\prime}=M_{e m}^{\prime}$,

$$
M_{e m}^{\prime}=2.29701 \times 10^{30} \mathrm{gms}=0.00116 M_{0}
$$

Here $M_{0}=1.99 \times 10^{33} \mathrm{gms}$ is the mass of sun.

\section{Conclusions}

In equation (47) to stop electromagnetic interaction the required mass is $2.29701 \times 10^{30} \mathrm{gms}\left(=0.00116 M_{0}\right)$. The mass of Jupiter planet is $1.898 \times 10^{30} \mathrm{gms}$ and the mass required to stop electromagnetic interaction is just 1.21 times greater than Jupiter's mass. So in any planet more than this mass life cannot survive, because in that planet electromagnetic interaction will be stopped by the gravity. Since the life is nothing but the low energy level electromagnetic interaction. Also this gives another interesting conclusion that more than the mass $0.00116 M_{0}$ (=384.63 times of earth's mass) there is no charged planets or stars. On the other hand when electromagnetic interaction stops by gravitational field then starts nuclear interaction and a star is born. This is the reason that stars are electrically neutral. Hence the metric given by the (75) is valid less than the aforesaid mass $2.29701 \times 10^{30} \mathrm{gms}$

\section{References}

[1] A. Einstein, "On the general theory of relativity", Annalen der physic, 49; 769-822 (1916). English translation in the "The principle of Relativity by Metheun, Dover publications (1923).

[2] K. Schwarzschild, "On the gravitational field of a point-mass according to Einstein theory", Sitzungsber. Preuss. Akad. Wiss. Phys. Math. Kl.; 189 (English translation) Abraham Zeimanov J, 1: 10-19 (1916).

[3] R. P. Kerr, "Gravitational field of a spinning mass as an example of algebraically special Metrics", Physical Review Letters, 11 (5): 237-240 (1963).

[4] E. Newman, A. Janis, "Note on the Kerr spinning-particle metric", Journal of Mathematical Physics 6 (6); 915-917 (1965). 
[5] J. B. Hartle, “Gravity-An introduction to Einstein's general relativity”, Pearson, 5th ed., p 400- 419, (2012).

[6] S. Weinberg, "Gravitation and Cosmology", Wiley India Private Ltd., p412-415 (2014).

[7] A. Z. Friedman, Phys. A, 10 (1), p377 (1922).

[8] H. P. Robertson, Astro. Phys. J., 82, p284 (1935).

[9] T. Kaluza, "On the unification problem in physics", Sitzungsber. Preuss. Akad. Wiss. Berlin (Math. Phys): 966$972(1921)$

[10] O. Klein, "The atomicity of electricity as a quantum theory law", Nature, 118: 516 (1926).

[11] O. Klein, "Quantum theory and five dimensional relativity", Zeit. F. Physik 37: 895, Reproduced in O'Raiferaigh's book (1926).

[12] A. S. Eddington, The mathematical theory of relativity", Published by Cambridge University Press; 185-187 (1923).

[13] H. Reissner, "Uber die eigengravitation des elektrischen felds nach der Einsteinschen theorie", Annalen der Physik (in German) 50 (9): 106-120 (1916).

[14] G. Nordstrom, "On the energy of the gravitational field in Einstein theory", Proc. Amsterdam Acad., 20, p1238 (1918).

[15] G. B. Jeffery, "The field of an electron on Einstein's theory of gravitation", Proceeding Royal Society, A99: 123-134 (1921).

[16] D. Finkelstein, "Past-future asymmetry of the gravitational field of a point particle", Physical Review 110: 965-968 (1958).
[17] R. H. Boyer and R. W. Lindquist, "Maximal analytic extension of the Kerr metric", J. Math. Phys. 8 (2): 265-281 (1967).

[18] E. Newman, E. Couch, K. Chinnapared, A. Exton, A. Prakash, R. Torrence, "Metric of a rotating Charged mass", Journal of Mathematical Physics, 6 (6): 918-919 (1965).

[19] L. Ryder, "Introduction to General Relativity" Cambridge university press, 265-271 (2019).

[20] N. Bijan, "Schwarzschild-like solution for ellipsoidal celestial objects”, Int. J. Phys. Sci. 6 (6): 1426-1430 (2011).

[21] R. J. Beach, "A classical Field Theory of Gravity and Electromagnetism", Journal of Modern Physics, 5: 928-939 (2014).

[22] M. D. Yu-Ching, A derivation of the Kerr metric by ellipsoid coordinates transformation", International Journal of Physical Science, 12 (11): 130-136 (2017).

[23] M. A. El-Lakany, "Unification of gravity and electromagnetism", Journal of Physical Science And Application, 7 (3): 15-24 (2017).

[24] L. J. Wang, "Unification of gravitational and electromagnetic forces", Fundamental Journal of Modern Physics 11 (1): 29-40 (2018).

[25] B. K. Borah, "Gravitational and electromagnetic field of an isolated proton", Journal of Ultra-scientist of Physical Science-A, 31 (3): 23-31 (2019).

[26] B. K. Borah, "Gravitational and electromagnetic field of an isolated positively charged particle", International Journal of Applied Mathematics and Theoretical Physics, 6 (4): 54-60 (2020). 